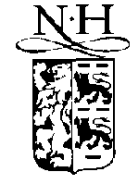

ELSEVIER

\title{
$r$-refinement for evolutionary PDEs with finite elements or finite differences
}

\author{
Paul A. Zegeling 1 \\ Computational Science Group. Department of Mathematics, University of Utrecht, P.O. Box 80.010, 3508 TA Utrecht, \\ The Netherlands
}

\begin{abstract}
In this paper two different moving-mesh methods ( $r$-refinement) are applied to evolutionary PDE models in one and two space dimensions. The first method (moving finite elements) is based on a minimization of the PDE residual that is obtained by approximating the solution with piecewise linear elements. The second method (moving finite differences) is based on an equidistribution principle with smoothing both in the spatial and the temporal direction. Theory predicts that the finite-element based moving-mesh method moves its grid points with the flow of the PDE, whereas the finite-difference based method moves its grid points with the steep parts of the PDE solution, respectively. Numerical experiments show some differences and similarities between the finite-element and finite-difference case when applied to 1D and 2D time-dependent models of the convection-diffusion-reaction type. 1998 Elsevier Science B.V.
\end{abstract}

\section{Introduction to $r$-refinement}

The class of adaptive methods can roughly be subdivided into three sub-classes. The first, characterized by the term $h$-refinement, consists of methods which add grid points in regions of high spatial activity and delete grid points in areas with almost constant solution behaviour. The second type of method, denoted by $p$-refinement, increases or decreases the order of approximation in appropriate regions of the domain. $r$-refinement methods, however, keep both the number of unknowns and the order of approximation constant, while moving the grid points in a dynamic way with the steep parts of the solution or other characteristic features of the underlying model. This paper treats two different methods from this class. Of course, it is possible and, in practice appropriate, to combine different types of methods, e.g., to work with $h-p-, h-r-, r-p-$, or even $h-p-r$-refinement.

During the last decade, $r$-refinement methods have been shown to be very useful for solving parabolic and hyperbolic partial differential equations involving fine scale structures such as steep moving fronts, emerging steep layers, pulses and shocks. In one space dimension moving-grid methods have been

\footnotetext{
'E-mail: zcgeling@math.ruu.nl. WWW: http://www.math.ruu.nl/people/zegeling/. 
applied successfully to a large class of PDE systems (see, e.g., $[2,5,6,9,10]$ ). In two space dimensions application of moving-grid methods is far less trivial than in 1D (see [3] however).

The first method in this paper is a moving-finite-element method (MFE) which is derived by minimizing the PDE residual obtained from the approximation of the solution with piecewise linear elements $[1,6]$. The second method is a moving-finite-difference method (MFD) which is defined by first transforming the PDE to its Lagrangian form and then coupling the discretized equations to the equidistribution based moving-grid equations. Numerical experiments show results for both the finiteelement and finite-difference case when applied to PDEs in $1 \mathrm{D}$ and 2D with convection, diffusion and reaction terms. Note that the intention of this paper is not to compare both methods, but rather to indicate the main differences and similarities in grid properties of MFE and MFD.

\section{2. $r$-refinement with finite elements}

Consider the following time-dependent PDE in $d$ space dimensions:

$$
\frac{\partial u}{\partial \ell}=\delta \Delta u-\beta \cdot \nabla u+S:=\mathcal{L}(u)
$$

for $\boldsymbol{x} \in \Omega \subset \mathbb{R}^{d}, t>0$, with $u=0$ on the boundary $\partial \Omega$ (for the purpose of this description) and initial condition for $t=0$. In (1) $\delta>0, \beta=\left(\beta_{1}, \ldots, \beta_{d}\right)^{\mathrm{T}}, S$ and $\mathcal{L}$ represent a diffusion parameter, a velocity field, a nonlinear source term and a general right-hand side PDE operator, respectively.

Approximate the PDE solution by

$$
u \approx U=\sum_{j=1}^{M} U_{j}(t) \alpha_{j}\left(\boldsymbol{x},\left\{\boldsymbol{X}_{k}(t)\right\}\right),
$$

where $M$ denotes the total number of grid points, and $\alpha_{j}$ are piecewise linear 'hat' functions on a time-dependent grid $\left\{\boldsymbol{X}_{k}(l)\right\}$.

Differentiating with respect to time $t$ and using the time-dependence of the grid points $\left(X_{1, j}(t), \ldots, X_{d, j}(t)\right)$, we obtain

$$
\frac{\partial U}{\partial t}=\sum_{j=1}^{M}\left\{\dot{U}_{j} \alpha_{j} \mid \sum_{l=1}^{n} \dot{X}_{l, j} \gamma_{l . j}\right\},
$$

where $\gamma_{l, j}=\partial U / \partial X_{l, j}$ and the dot stands for $\mathrm{d} / \mathrm{d} t$. The equations determining the semi-discrete unknowns $U_{j}, X_{1, j}, \ldots, X_{d . j}$ are obtained by minimizing the PDE residual $\mathcal{R}$ without restriction to $\dot{U}_{i}, \dot{X}_{1, i}, \ldots, \dot{X}_{d, i}$, where

$$
\mathcal{R}:=\left\|\frac{\partial U}{\partial t}-\mathcal{L}(U)\right\|_{L_{2}(\Omega)}^{2}+\mathcal{Q}^{2} .
$$

The second term $\mathcal{Q}^{2}$ is a regularization term containing small parameters $\varepsilon_{1}^{2}$ and $\varepsilon_{2}^{2}$ to prevent the parameterization (3) from becoming degenerate (for more details see $[6,10]$ or remark below about mass-matrix). The minimization yields the stiff ODE system

$$
\mathcal{M}_{1}\left(\zeta, \varepsilon_{1}^{2}\right) \dot{\zeta}=H_{1}\left(\zeta, \varepsilon_{2}^{2}, \delta\right)
$$


with $\zeta=\left(\ldots, U_{j}, X_{1, j}, \ldots, X_{d, j}, \ldots\right)^{\mathrm{T}}$ and an initial vector $\zeta(0)$ containing the initial solution vector $U(0)$ and the initial grid vector $X_{d}(0)$. In (5) $\mathcal{M}_{1}$ is an extended mass-matrix, which is strictly positive-definite for $\varepsilon_{1}^{2}>0$, but may be singular when $\varepsilon_{1}^{2}=0$. The choice for the two regularization parameters $\left(0<\varepsilon_{1}^{2} \ll 1,0<\varepsilon_{2}^{2} \ll 1\right)$ is not essential for this paper, in which we describe the grid properties, although it would be of importance for more general applications. In the numerical examples the values are $\varepsilon_{1}^{2}=10^{-4}$ and $\varepsilon_{2}^{2}=10^{-10}$.

For $d=1$, it can be shown that the grid points in the semi-discrete equations (5) in the limit $(M \rightarrow \infty)$ satisfy

$$
\dot{x}=\beta+\delta\left[2 \frac{u_{x x x}}{u_{x x}}-3 \frac{\xi_{x x}}{\xi_{x}}\right],
$$

where $\xi$ is a transformed space variable (see, e.g., [10]). In steady-state situations it can be derived from (6) that the grid is equidistributed according to the rule $\left(x_{\xi} \widehat{W}\right)_{\xi}=0$, with a weight-function $\widetilde{W}=\left|u_{x}\right|^{1 / 3}\left|u_{x x}\right|^{2 / 3}$. This indicates that grid points are expected to be concentrated in regions of large second derivatives.

In contrast with the 1D situation no theory is available to predict such an 'equidistributional' behaviour in the two dimensional case. However, it is conjectured that for $d=2$, without regularization terms (i.e., with $\varepsilon_{1}^{2}=\varepsilon_{2}^{2}=0$ ), the grid movement satisfies

$$
\dot{x}-\beta_{1}+\delta \phi_{1}, \quad \dot{y}-\beta_{2}+\delta \phi_{2},
$$

with functions $\phi_{1}$ and $\phi_{2}$ depending on spatial derivatives. Note that (7) is a property of MFE and not the definition of the grid motion. For more details on this method and more sophisticated implementations, see, e.g., [1,3].

\section{3. $r$-refinement with finite differences}

A moving-finite-difference method in two space dimensions $(d=2$, see also [11]) is obtained by first transforming the PDE ( 1 ) to a PDE in a moving frame using a coordinate transformation $x=x(\xi, \eta, \theta), y=y(\xi, \eta, \theta), t=\theta$. This gives the Lagrangian form of the PDE

$$
\dot{u}-u_{x} \dot{x}-u_{y} \dot{y}=\mathcal{L}(u),
$$

where the dot stands for $\partial / \partial \theta$. Defining a time-dependent spatial grid $\left(\left\{X_{i}(t)\right\},\left\{Y_{i}(t)\right\}\right)$ the Lagrangian form is semi-discretized using central differences for the spatial derivatives:

$$
\dot{U}_{i}-U_{x, i} \dot{X}_{i}-U_{y, i} \dot{Y}_{i}=L_{i},
$$

where $L_{i}$ is the discretized form of $\mathcal{L}$ on a non-uniform grid. To complete the ODE system, the following moving-grid PDEs are semi-discretized:

$$
\frac{\partial}{\partial \xi}\left[\frac{\tilde{n}+\tau \dot{\tilde{n}}}{V}\right]=0, \quad \frac{\partial}{\partial \eta}\left[\frac{\tilde{m}-\tau \dot{\tilde{m}}}{W}\right]=0,
$$

where the weight functions are

$$
V:=\sqrt{1+\alpha u_{x}^{2}}, \quad W:=\sqrt{1+\alpha u_{y}^{2}},
$$


and the point concentrations (along coordinate lines) are $n:=1 / x_{\xi}$ and $m:=1 / y_{n}$.

In (8) $\tilde{n}$ represents a smoothed point concentration

$$
\tilde{n}:=\left(I-\frac{\kappa(\kappa+1)}{M} \frac{\partial^{2}}{\partial \xi^{2}}\right) n,
$$

where $\kappa$ is a spatial smoothing parameter ( $\widetilde{m}$ is defined similarly in the $y$-direction) and $I$ is the identity operator. Loosely speaking, the weight functions $V$ and $W$ determine the shape of the grid distribution and $\kappa$ the level of clustering. Eq. (8) describes a two-dimensional equidistribution principle with smoothing. With $\kappa=2$ rather modestly graded space grids are obtained. The parameter $\tau$ in (8) is connected with the temporal grid smoothing and serves to act as a tool for suppressing grid oscillations in time (for more details, see [10]). The parameter $\alpha$ controls the level of adaptivity. Semi-discretization of (8) combined with the discretized Lagrangian PDE gives a stiff system of ODEs

$$
\mathcal{M}_{2}(\zeta, \tau) \dot{\zeta}=I_{2}(\zeta), \quad t>0
$$

with $\zeta$ defined as for the MFE method.

In the following two sections numerical experiments are described using the moving grid methods defined by (5) and (9). Both MFE and MFD define a stiff ODE system with a banded matrix that can be solved using the ODE package DASSL [8]. In all cases, we have used a time-tolerance $\mathrm{TOL}=10^{-3}$ for DASSL, a uniform starting grid $\left(\left\{X_{i}(0)\right\},\left\{Y_{i}(0)\right\}\right)$, and a 'standard' choice for the method parameters, i.e., $\alpha=1, \kappa=2, \tau=10^{-3}$ for MFD (see previous section for MFE parameters). Here, 'standard' means that this parameter choice could generally be used if the PDE is well-scaled in time and space, e.g., $\Omega=[0,1]^{2}$ and the critical time-scale of the problem is $t \in[0, \mathcal{O}(1)]$.

\section{Numerical examples in 10}

In 1D the semi-discrete MFE and MFD equations can be deduced from (5) and (9) by restricting all operators and discretizations to one space dimension.

To show the usefulness of $r$-refinement (even in 1D) the following example is given (on a fixed uniform grid several thousand grid points would be required to obtain similar accuracy!):

Example 1. The Gray-Scott reaction-diffusion system for two irreversible chemical reactions " $\mathcal{U}+$ $2 \mathcal{V} \rightarrow 3 \mathcal{V}, \mathcal{V} \rightarrow \mathcal{P}^{\prime \prime}$ is defined by

$$
\frac{\partial u}{\partial t}=\Delta u-u v^{2}+A(1-u), \quad \frac{\partial v}{\partial t}=\delta^{2} \Delta v+u v^{2}-B v .
$$

Here $A$ stands for the rate at which $\mathcal{U}$ is fed from the reservoir into the reactor and $k_{2}(:=B-A)$ for the rate at which $\mathcal{V}$ is converted to an inert product $\mathcal{P}$. As an example, $\mathcal{U}$ may represent 'ferrocyanide' or 'sodium hydroxide', and $\delta^{2}$ a non-dimensionalized diffusivity constant of the chemicals. In Fig. 1 the grid history (401 grid points) and the solution at $t=2400$ are given for MFD. A smooth grid, both in time and in space, is obtained which follows the important features in this model. For more details about this application we refer to [4]. 

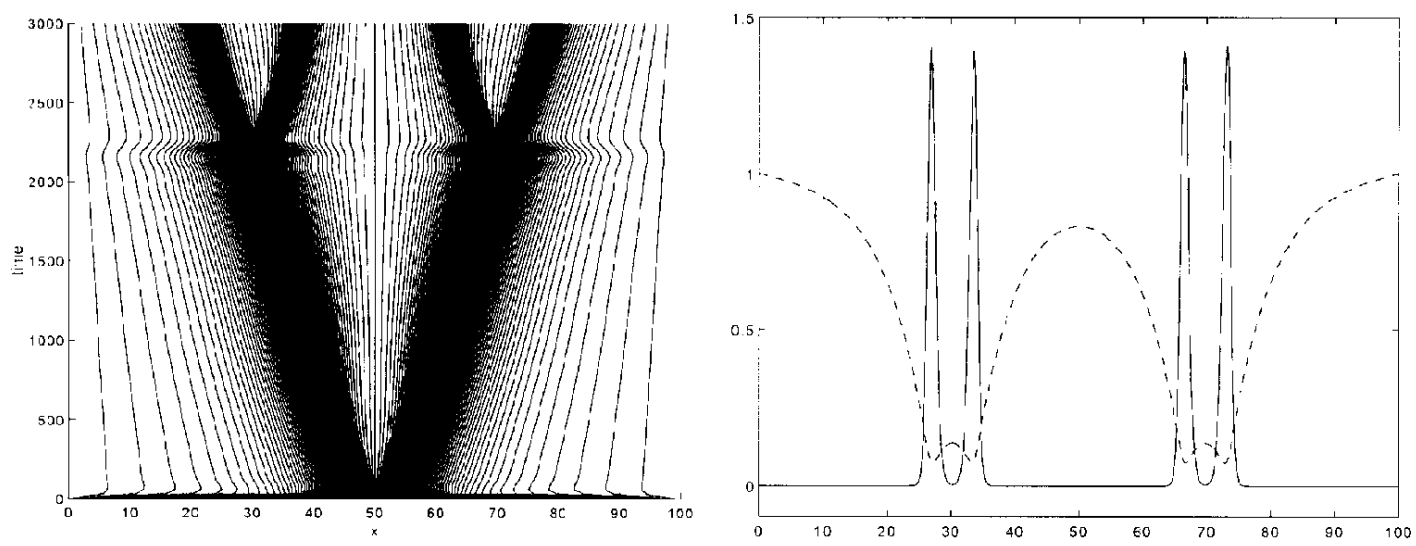

Fig. 1. Moving-finite-difference results for the 1D reaction-diffusion system (10). Left: grid history, and right: solutions at $t=3000(--: u$ and $-: v)$.

To show the main difference in behaviour of the two described $r$-refinement methods it is illustrative to look at the following simple model:

\section{Example 2.}

$$
\frac{\partial u}{\partial t}+\frac{\partial u}{\partial x}=0,\left.\quad u\right|_{x=0}=\left.u\right|_{x=1},\left.\quad u\right|_{t=0}=[\sin (\pi(x+0.3))]^{50}
$$

The exact solution, $u(x, t)=[\sin (\pi(x-t+0.3))]^{50}$, is a moving pulse with constant velocity that penetrates the right-hand side boundary and re-appears at the left boundary, due to the periodic boundary conditions. We know from the theoretical properties that MFE will follow the pulse with almost exact speed, while keeping the shape of the pulse without any numerical disturbance (cf. (6) with $\delta=0$ ). However, at $t=0.6$, we see in Fig. 2 that the method tries to move its grid points through the boundary, not recognizing the 'new' pulse at the left boundary and therefore collapses at the right boundary. In the same figure (on the right) the corresponding grid is given for MFD. This method follows the solution derivatives smoothly and therefore recognizes the newly-formed pulse very rapidly. It is also seen that after $t=1$ the MFD grid is much less smooth and somewhat 'diffused'.

\section{Numerical examples in $2 \mathrm{D}$}

In two space dimensions the effects of collapsing grids can even be stronger. Consider the following hyperbolic PDE, which is a popular model for testing adaptive and non-adaptive numerical schemes [7]:

\section{Example 3.}

$$
\frac{\partial u}{\partial t}+\beta \cdot \nabla u=0, \quad \nabla \cdot \beta=0
$$



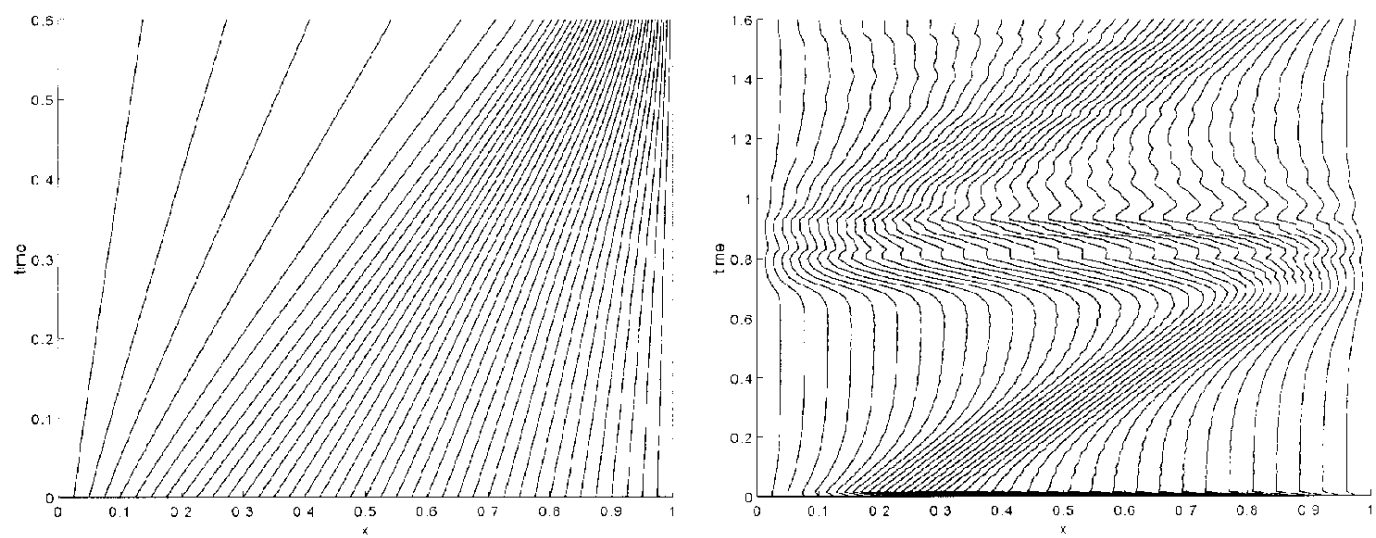

Fig. 2. Moving-grid results for Example 2; MFE (left) versus MFD (right). Note the difference in time-scales: the left picture is zoomed in to show the collapsing grid points at the right boundary more clearly.

with $\beta=\pi\left(y-\frac{1}{2} ; \frac{1}{2}-x\right)^{\mathrm{T}}, \Omega=[0,1]^{2}$, and

$$
\left.u\right|_{\partial \Omega \Omega}-0,\left.\quad u\right|_{t=0}=\mathrm{e}^{-200\left((x-0.5)^{2}+(y-0.65)^{2}\right)} .
$$

The exact solution, $u(x, y, t)=\mathrm{e}^{-200\left((x-r(t))^{2}+(y s(t))^{2}\right)}$ (with $r(t)=(2+\sin (\pi t)) / 4$ and $s(t)=$ $(2+\cos (\pi t)) / 4)$, describes a pulse that moves around in circles with a constant speed. During this movement the shape of the pulse does not change. It is known that on a non-moving grid either the solution is damped out rapidly due to numerical diffusion or unnatural oscillations may appear. In Fig. 3 test results are displayed using MFE and MFD. Following the flow in the model (cf. (7) with $\delta=0$ ), MFE tends to twist the grid structurc. The solution accuracy is very high however: the contourlines remain almost undisturbed during the time-integration. It should be noted that reconnecting the grid points may only help the method temporarily, since the circular motion of the grid points remains throughout the time-integration.

For MFD less grid distortion occurs: the grid points now follow the gradients of the solution. For later points of time (e.g., $t=1.0$ ) a slight instability in the grid motion seems to arise, yielding a more 'skewed' grid than for $t=0.5$. This strange phenomenon should still be investigated further. Regridding combined with mesh movement ( $h-r$-refinement) may be beneficial for these types of models, at least for MFD.

To show the "equidistribution-like' behaviour of the MFE grid in 2D, in Fig. 4 MFE results are shown at two different points of time for the $2 \mathrm{D}$ version of the reaction-diffusion model (10). Now we sec, in contrast with the hyperbolic model, a nice positioning of grid points near steep transitions in the solution without grid distortion (cf. (7) with $\beta=(0,0)^{\mathrm{T}}$ ). We predict a similar behaviour for general reaction-diffusion systems in 'any' space dimension.

\section{Conclusions}

The finite-element based $r$-refinement method, MFE, for which a formulation is possible in 'any' space dimension, follows the flow (PDE characteristics) in the model. For reaction-diffusion PDEs 

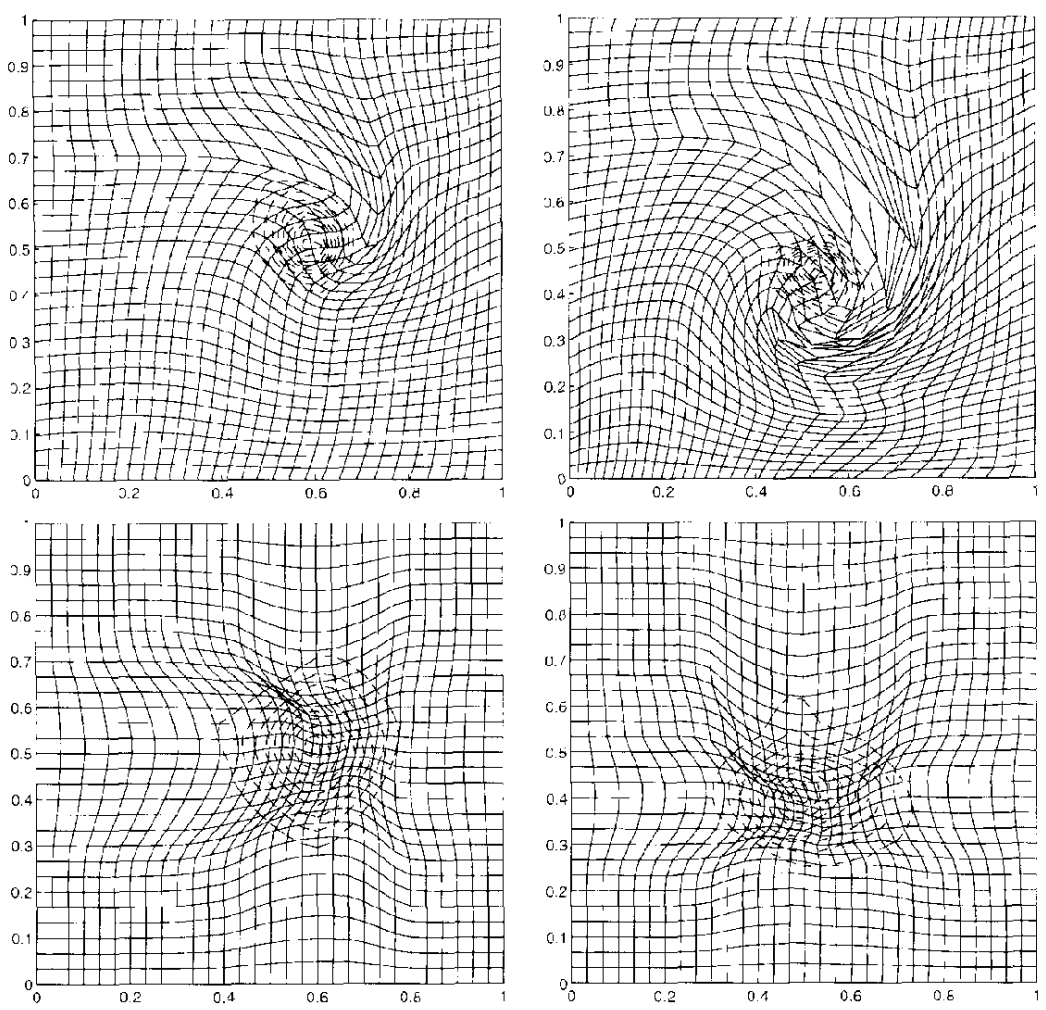

Fig. 3. Moving-grid results for Example 3 at $t=0.5$ and $t=1$; MFE (top) versus MFD (bottom).
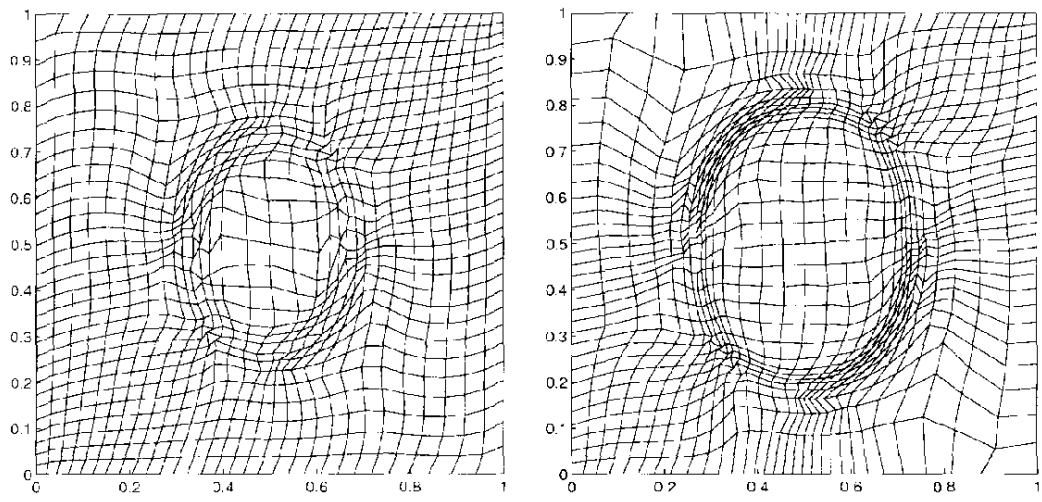

Fig. 4. MFE grid for the 2D reaction-diffusion system (10) at $\ell=20$ (left) and $\iota=50$ (right).

it produces smooth adaptive grids without distortion (following now the solution characteristics). On the other hand, for PDEs with strong convection terms accurate solutions can be expected, however on possibly twisted grids.

The finite-difference based $r$-refinement method, MFD, can be formulated in one and two space dimensions, and follows the steep parts of the PDE solution itself. Satisfactory results are obtained on 
simple test problems in 2D. There is room to improve the underlying moving-mesh PDEs with respect to grid quality and numerical efficiency in solving the semi-discrete ODE system.

As a recommendation, it could be stated that MFD may be used to efficiently approximate solutions of convection-diffusion-reaction systems in one space dimension having steep moving transitions (in 2D the method needs to be investigated further before drawing any final conclusion), and MFE may be used for reaction-diffusion systems in 'any' dimension (one should be aware of possible grid-distortion when first-derivative terms are present in the PDE model though).

In general applications it could be beneficial to define an $h-r$-refinement technique for both of the described methods in order to deal with possible grid de-generations.

\section{References}

[1] M.J. Baines, Moving Finite Elements (Clarendon Press, Oxford, 1994).

[2] N. Carlson and K. Miller, Design and application of a gradient-weighted moving finite element code, Part I, in 1D, Technical Report 236, Purdue University, West Lafayette, IN (1994).

[3] N. Carlson and K. Miller, Design and application of a gradient-weighted moving finite element code, Part II, in 2D, Technical Report 237, Purdue University, West Lafayette, IN (1994).

[4] A. Doelman, T.J. Kaper and P.A. Zegeling, Pattern formation in the one-dimensional Gray-Scott model, Nonlinearity 10 (1997) 523-563.

[5] W. Huang, Y. Ren and R.D. Russell, Moving mesh partial differential equations (MMPDEs) based on the equidistribution principle, SIAM J. Numer. Anal. 31 (3) (1994) 709-730.

[6] K. Miller and R.N. Miller, Moving finite elements I, SIAM J. Numer. Anal. 18 (1981) 1019-1032.

[7] C.R. Molenkamp, Accuracy of finite-difference methods applied to the advection equation, J. Appl. Meteorol. 7 (1968) $160-167$.

[8] L.R. Petzold, A description of DASSL: a differential/algebraic system solver, in: R.S. Stepleman, ed., IMACS Transactions on Scientific Computation (1983).

[9] P.A. Zegeling, J.G. Verwer and J.C.H. van Eijkeren, Application of a moving-grid method to a class of 1D brine transport problcms in porous media, Internat. J. Numer. Methods Fluids 15 (2) (1992) 175-191.

[10] P.A. Zegeling, Moving-grid methods for time-dependent partial differential equations, CWI Tract No. 94, Centre for Mathematics and Computing Science, Amsterdam (1993).

[11] P.A. Zegeling, A dynamically-moving adaptive grid method based on a smoothed equidistribution principle along coordinate lines, in: B.K. Soni, J.F. Thompson, J. Häuser and P. Eiseman, eds., Proc. 5th International Conference on Numerical Grid Generation in Computational Computational Field Simulation, Starkville (1996). 